\title{
Beiträge zur Lehre von der Entstehung neuer Gewohnheiten bei den Tieren.
}

\author{
Von \\ Dr. J. S. Szymanski (Wien). \\ (Aus dem physiologischen Institut der Üniversität Wien ${ }^{1)}$ ). \\ (Mit 8 Textabbildungen.) \\ Inhalts übersicht: \\ I. Lernversuche an Fröschen . . . . . . . . . . . . . . . . . 125 \\ II. Über das Verklingen einer früher erworbenen Gewohnheit. . . . . 129 \\ IJI. Ein Versuch über die Fähigkeit der Tiere, Erfabrungen zu verallgemeinern 137 \\ IV. Die Abhängigkeit der Lerngeschwindigkeit von der Antriebsstärke . . . 141 \\ V. Lin Fall des denkbar vollkommensten Verlaufes eines Lernvorganges. . 148
}

\section{Lernversuche an Fröschen ${ }^{2}$ ).}

Die Methode, mit der ich die Lernfähigkeit der Frösche untersucht habe, war die folgende:

Eine geräumige Glaswanne $A B$ war mit einer ca. 0,25\% igen Formalinlösung ${ }^{3}$ ) gefüllt. Daranfhin wurde ein an beiden Enden offener Zylinder (ea. $30 \mathrm{~cm}$ lang, $11 \mathrm{~cm}$ im Durchmesser) aus feinem Drahtnetz $(C D)$ in die Wanne derart eingetaucht, dass derselbe mit einer Längswand und einem offenen Ende $C$ an den Wannenwänden dicht anlag. Um das Hin- und Herrollen des Zylinders zu verhindern, waren zwei grosse mit Wasser vollgefüllte Flaschen $M$ in den freien Raum zwischen dem Zylinder und der gegenüberliegenden Wand angebracht. Auf der oberen Mantelfläche des Zylinders, gerade in der

1) Herrn Prof. Dr. A. Kreidl gebührt wie immer mein besonderer Dank für die tatkräftige Unterstützung meiner wissenschaftlichen Bestrebungen.

2) Die Lernfähigkeit der Frösche bzw. der Kröten wurde von Yerkes, Sch äffer und Buytendijk geprüft.

3) Auf 12 Liter Wasser $30 \mathrm{ccm}$ Formalin. 
Mitte seiner Länge, befand sich ein Fenster $(F)$, in das ein Glasrohr von einem mit dem Fenster gleichen Durchmesser (ca. 7,5 cm) $(E F)$ eingeführt wurde (Abb. 1).

Der in das Glasrohr eingeführte Froseh sollte nun erlernen, zwischen dem offenen und abgesperrten Ausgang zu unterscheiden und sich der Wirkung der Formalinlösung zu entziehen.

Der genaue Verlauf einer. Versuchsserie war folgender:

Der Frosch wurde in den Vorhof (Glasrohr) eingeführt. Falls er bei der Berührung mit der Formalinlösung kehrtmachte und das Glasrohr hinaufklettern wollte, wurde ihm das Entschlüpfen durch das Einführen eines Korkstöpsels, der auf einem Stab befestigt war und den gleichen Durchmesser mit dem Glasrohr hatte, verwehrt.

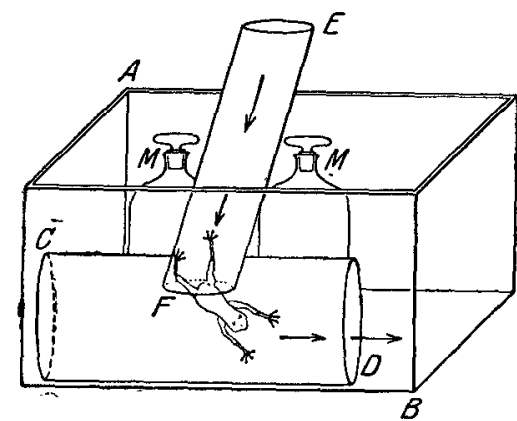

Abb. 1. (Erklärung im Text.) Nachdem der Froseh den Zylinder nach einigen Umwegen durch den offenen Ausgang verlassen hatte, wurde derselbe mit einem kleinen Netz schnell herausgefischt, in tine bereitstehende Wanne mit reinem Wasser eingetaucht und schliesslich in ein kleines Aquarium gesetzt. Nach einer Rubepause von 1-2 Minuten wurde wiederum der Versuch wiederholt usf. Dies dauerte so lange (ca. 1 bis höchstens 2 Stunden), bis der Frosch den offenen Ausgang in den zehn aufeinanderfolgenden Versuchen auf dem kürzesten Wege aufgefunden hat.

Bei jeder Versuchsserie wurde die Temperatur der Formalinlösung bestimmt: sie schwankte in sämtlichen Versuchen zwischen $13,4-16,8^{\circ} \mathrm{C}$.

Falls ein Versuchsfrosch zu Beginn einer Versuchsserie eine Neigung zeigte, stets gegen den offenen Ausgang zu schwimmen, wurde der letztere abgesperrt, der andere geöffnet, und die Versuche von neuem begonnen. Dies letztere war der Fall bei den Fröschen Nr. 2, 7, 8.

13 Frösche, von denen Nr. 1 bis einschliesslich Nr. 8 Seefrösche, Nr. 9 bis einschliesslich Nr. 13 Wasserfrösche waren, wurden mit Erfolg untersucht; mit einigen weiteren Tieren konnten die Versuche wegen der grossen Erschöpfung der betreffenden Indi- 
Beiträge zur Lehre von der Entstehung neuer Gewohnheiten bei den Tieren. 127

viduen - ich arbeitete nämlich mit Winterfröschen - nicht zu Ende geführt werden.

Bei den Versuchen an den Tieren Nr. 1, 3, 5, 10, 12 war der linke Ausgang offen, der rechte ahgesperrt; bei den Versuchen an den Fröschen Nr. 4, 6, 9, 11, 13 war das Umgekehrte der Fall. Bei den Versuchen an den Tieren Nr. 2, 7, 8, war zunächst der linke und daraufhin der rechte Ausgang offen.

Die Versuchsergebnisse sind grapbisch in der Abb. 2 (S. 128) dargestellt.

Aus den Kurven der Abb. 2 ergibt sich, dass, wenn man von einem Fall einer ausserordentlich kurzen Lernzeit (Nr. 9 - nach fünf Versuchen) und von vier Fällen einer recht langen Lernzeit (Nr. 1, 8, 10, 11 - nach 35-61 Versuchen) absieht, die überwiegende Mehrzahl der Frösche (Nr. 2, 3, 4, 5, 6, 7, 11, 12) nach durchschnittlich 15 Versuchen (beide Grenzfälle waren 10 und 21 Versuche) zwischen dem abgesperrten und offenen Ausgange zu unterscheiden lernte.

Mit der fortschreitenden Übung sank auch die Zeit, die vom Momente des Einsetzeus des Frosehes ins Glasrohr bis zum Momente des Verlassens des Zylinders durch den offenen Ausgang verstrich; so war zum Beispiel diese Zeit bei dem Frosch Nr. 3, der wohl als typischer Fall gelten darf, die folgende:

\begin{tabular}{c|c}
\hline Nummer des Versuches & Sekunden \\
\hline 1 & 117 \\
2 & 1 \\
3 & 32 \\
4 & 18 \\
5 & 1 \\
6 & 29 \\
7 & 53 \\
8 & 1 \\
9 & 41 \\
10 & 19. \\
11 & 43 \\
12 & 32 \\
13 & 47 \\
14 & 1 \\
15 & 80 \\
$16-25$ & 1
\end{tabular}

Die Ergebnisse dieser Versuche berechtigen zu dem Schluss, dass selbst die wenig sensiblen Tiere bei einem genügend starken Antrieb fähig sind, eine relativ einfache Handlung relativ schnell zu erlernen. 

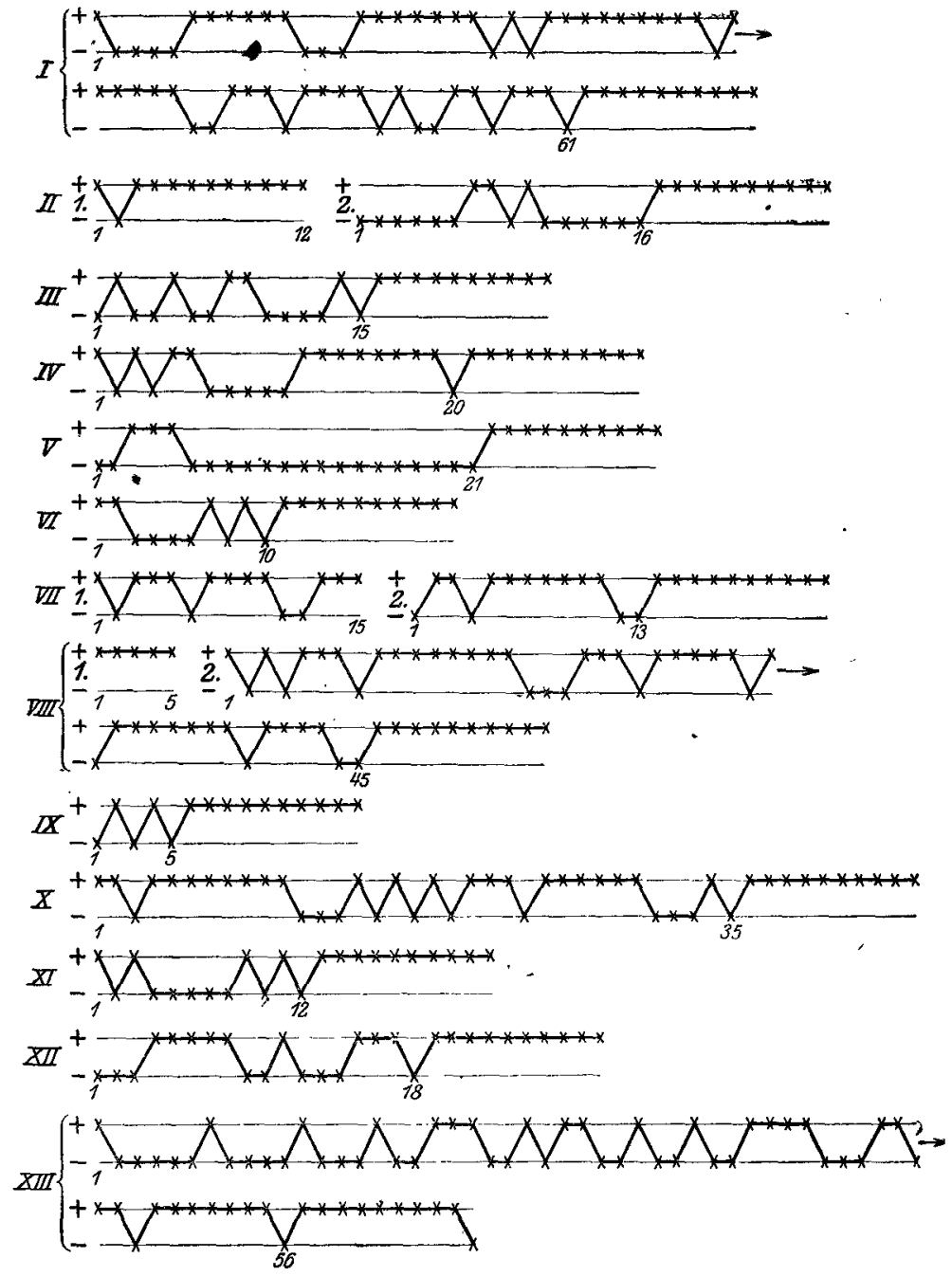

Abb. 2. Auf der + Linie sind die Fälle eingetragen, in denen der Froseh den offenen Ausgang auf dem kürzesten Wege erreicht hat; auf der - Linie sind jene Fälle eingetragen, in dewen der Frosch zunächst gegen den abgesperrten Ausgang hinschwamm, um erst nachher den offenen zu finden. Die Kreuze bedeuten die aufeinanderfolgenden Versuche; die Zahl unten zeigt, nach wievieltem Versuche der Frosch die von ihm verlangten zehn aufeinanderfolgenden richtigen Handlungen ausgeführt hat. - Bemerkung zur Kurve XIII: Der Frosch Nr. 13 schlug zuletzt neunmal die richtige und das zehntemal die falsche Richtung ein. Angesichts dessen, dass dieser Frosch starke Ermüdungserscheinungen (Schlafstellung und volle Regungslosigkeit in den Zwischenpausen) aufwies, dass derselbe bei dem letzten Versuche sich überhaupt nicht bewegen wollte und erst durch das wiederholte Stossen zur Bewegung gebracht werden konnte, und schliesslich angesichts dessen, dass dieser Frosch in den 18 letzten Versuchen 16 mal richtig handelte, habe ich das betreffende Versuchsresultat als positiv angesehen. - Bemerkung zur Kurve $I$ : Die lange Lernzeit dieses Tieres steht vielleicht damit im Zusammenhang, dass dieser Frosch aus Versehen in einer bloss $0,08 \%$ Formalinlösung untersucht worden war. 
Beiträge zur Lebre von der Entstehung neuer Gewohnheiten bei den Tieren. 129

\section{Ubber das Verklingen einer früher erworbenen Gewohnheit.}

In einer früberen Untersuchung habe ich einen Apparat (Mnemograph) beschrieben, der den Lernvorgang bei weissen Mäusen automatisch zn registrieren vermochte ${ }^{1}$ ).

Die gleiche Methode schien mir geeignet zu sein, um den Vorgang des Verklingens einer erworbenen Gewohnheit zu untersuchen.

Für diesen Zweck war der Apparat derart abgeändert, dass statt eines Futtertisches und zwei hinzuführenden Brücken bloss zwei in der Entfernung von $10 \mathrm{~cm}$ parallel zueinander lagernde $15 \mathrm{~cm}$ lange Fattertische angebracht waren.

Jeder Futtertisch bestand aus einer Leiter $E$ und einer eigentlichen Tischplatte $A B$ mit eirem Futternapf $C$ (Abb. 3, I).

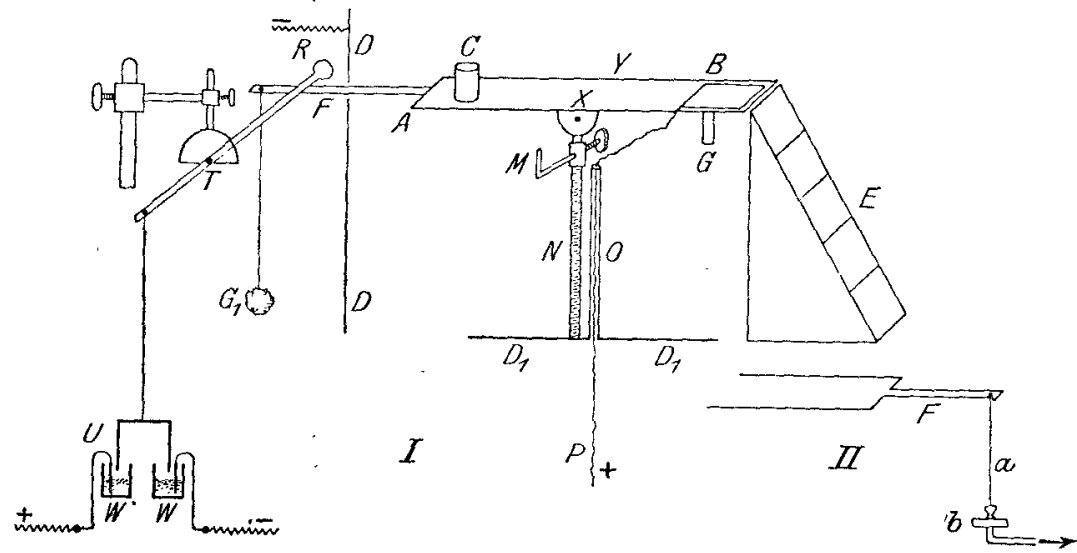

Abb. 3. (Erk]ärung im Text.)

An das der Leiter entgegengesetzte Ende der Tischplatte war ein schmaler Aluminiumstreifen $F$ befestigt, der durch einen Spalt in der Käfigwand $D D$ nach aussen herausragte.

Die Tischplatte kounte um die Achse $x y$ fast reibungslos schwingen; durch das Gewicht $G$ und Gegengewicht $G_{1}$ (ein an einem Faden herabhängendes Stück Wachs) konnte die Tischplatte in labiles Gleichgewicht gebracht werden. Bei den Versuchen wurde das Gegengewicht $G_{1}$ etwas grösser als das Gewicht $G$ gemacht, so dass der

1) „Untersuchungen über den biologisch richtigen Verlauf des Lernvorganges bei weissen Mäusen“ (Pflüg er's Arch. Bd. 169 S. 537. 1917).

Pfláger's Archiv für Physiologie. Bd. 173. 
Streifen $F$ dem unteren Ende des Wandspaltes anlag. Bei der leisesten Berührung des Plattenendes $B$ senkte sich dieses Eade, und der Streifen $F$ hob sich, um beim Nachlassen des Druckes wieder ins Gleichgewicht $\mathrm{zu}$ kommen. Durch die Vorrichtung $M$ konnten die Schwingungen der Tischplatte bei dem Druck auf $B$ bis auf einige Millimeter beschränkt werden.

Auf dem der Leiter zugekehrten Tischende wurde eine isolierte Metallplatte $B$ angebracht. Ein Leitungsdraht $(P)$, der durch ein Glasrohr $O$ geführt war, verband diese Platte mit einem Pol der sekundären Spule von einem Induktionsapparat. Als zweiter Pol dienten die beiden Leiter und die Käfigwand $(R D)$. Bei dieser Anordnung musste das Versuchstier bei gleichzeitiger Berührung der Leiter $E$ und der Platte $B$ einen elektrischen Schlag bekommen. Bei dem offenen Tische wurde der Stromschluss dadurch vollzogen, dass ein Arm eines Hebels $T$, dessen zweiter Arm dem Streifen $F$ anlag, beim Betreten der Platte $B$ sich senkte. Infolgedessen tauchte eine Kupfergabel $U$, die mittels eines Fadens am Hebelarm befestigt war, in untergestellte Näpfe mit Quecksilber $W$ ein, die nit der primären Spule eines Induktionsapparates mit einem elektromagnetischen Schreiber und einem Akkumulator verbunden waren. Bei rem Nachlassen des Druckes uf $B$ senkte sich der Hebelarm $R$, die Gabel $U$ wurde dadurch aus den Quecksilbernäpfen herausgezogen und der Kontakt unterbrochen. Das Einschalten des Elektromagneten in den Stromkreis ermöglichte das Registrieren der jedesmaligen Berührung der Platte $B$ auf einem Kymographion mit 24 stündiger Umlaufszeit.

Um auch bei dem gesperrten Tische, was durch die Vorrichtung $M$ bewerkstelligt werden kounte, die Berührungen der Platte durch ein Versuchstier aufschreiben zu lassen, wurde die Vorrichtung $M$ jedesmal derart eingestellt, dass die Berührung des Tischendes $B$ die Senkung desselben um einige Millimeter bewirkte. Da bei dem ab. gesperrten Tische der Streifen $F(A b b .3 I I)$ mittels eines Fadens $a$ mit einer Aufnahmetrommel verbunden war, konnte die jedesmalige Berührung des Tisches aufgeschrieben werden.

Der Verlauf einer Versuchsserie war nun folgender:

Nachdem beide Tische abgesperrt, also für das Versuchstier, eine weisse Maus, zugänglich gemacht worden wąren, wurden beide Futternäpfe mit Futter gefüllt; daraufhin wurde in den Käfig ein Wattebausch (für das Nest!) und ein Wassernapf gegeben. Schliesslich 
Beiträge zur Lehre von der Entstehung neuer Gewohnheiten bei der Tieren. 131

wurde die Maus in den Käfig gesetzt. Nach Verlauf von 24 Stunden wurden die Kurven abgenommen und die Anzahl der Striche (Berübrungen der Tische!) auf beiden Kurven aufgezählt (Kontrollversuch). Daraufhin wurde jener Tisch, den die Maus während des Kontrollversuches relativ öfters berührt hat, aufgemacht und der Induktionsapparat eingeschaltet; der andere wurde abgesperrt, also für die Maus zugänglich gelassen. Die beiden Leitern und die Tischplatten wurden mit Äther abgewasichen, die Wasser- und Futternäpfe nachgefüllt. Nach 24 Stunden wurde wieder die Kurve abgenommen, die Striche aufgezählt, die Näpfe nachgefüllt und die Leitern und die Tische abgewaschen; die sonstige Versuchsanordnung blieb unverändert usf. Dies wiederholte sich täglich, , bis schliesslich die Maus einige Tage hindurch den offenen Tisch nicht mehr berührt hat. Hiermit nahmen die Lernversuche das Ende.

Fortab wurden beide Tische abgespert und die Versuche bei gleichbleibender sonstiger Versuchsanordnung fortgesetzt, bis die Maus nach eiuer Reihe von Tagen den früher offen gewesenen Tisch wieder betreten hat. Daraufhin wurde die ganze Versuchsserie nach Verlauf von einigen weiteren Tagen abgeschlossen.

Vier weisse Mäuse wurden auf diese Weise untersucht; die Versuchsanordnung wich bloss bei Nr. 1 von der oben beschriebenen insofern ab, als der Tisch, den die Maus zu vermeiden erlernen sollte, bei den Versuchen mit dieser Maus bis zum 15. Versuchstage offen, jedoch ohne das Einschalten vom Induktionsapparat, blieb; erst vom 15. Versuchstage an kam dieser Apparat zur Verwendung.

Die nächstfolgende Tabelle gibt die Versuchsergebnisse wieder.

\begin{tabular}{|c|c|c|c|c|c|}
\hline \multirow{2}{*}{$\begin{array}{l}\text { Num- } \\
\text { mer } \\
\text { des } \\
\text { Tieres }\end{array}$} & \multirow{2}{*}{$\begin{array}{l}\text { Ver- } \\
\text { suchs- } \\
\text { tage }\end{array}$} & \multirow{2}{*}{ Versuchsanordnung } & \multicolumn{2}{|c|}{$\begin{array}{c}\text { Anzahl } \\
\text { der Beribhrungen }\end{array}$} & \multirow{2}{*}{ Bemerkungen } \\
\hline & & & $\begin{array}{l}\text { Linker } \\
\text { Tiseh } \\
(=1 . \text { T. })\end{array}$ & $\begin{array}{l}\text { Rechter } \\
\text { 'Tisch } \\
(=\mathrm{r} \text {. T. })\end{array}$ & \\
\hline $\begin{array}{l}1 . \\
q\end{array}$ & $\begin{array}{l}1 . \\
2 . \\
3 . \\
4 . \\
5 . \\
6 . \\
7 . \\
8 . \\
9 . \\
10 . \\
11 . \\
12 . \\
13 . \\
14 .\end{array}$ & $\begin{array}{l}\text { Beide Tische - abgesperrt } \\
\text { r. T. = abgesp.; l. I'. = offen } \\
\text { dgl. } \\
\text { dgl. } \\
\text { dgl. } \\
\text { dgl. } \\
\text { dgl. } \\
\text { dgl. } \\
\text { dgl. } \\
\text { dgl. } \\
\text { dgl. } \\
\text { dgl. } \\
\text { dgl. } \\
\text { dgl. }\end{array}$ & $\begin{array}{r}42 \\
15 \\
5 \\
10 \\
9 \\
7 \\
0 \\
4 \\
0 \\
0 \\
4 \\
7 \\
5 \\
9\end{array}$ & $\begin{array}{r}21 \\
15 \\
33 \\
38 \\
87 \\
64 \\
76 \\
41 \\
129 \\
28 \\
53 \\
79 \\
67 \\
56\end{array}$ & $\begin{array}{l}\text { Der Schlitten- } \\
\text { apparat aus- } \\
\text { geschaltet }\end{array}$ \\
\hline & & & & & \\
\hline
\end{tabular}


Fortsetzung der Tabelle.

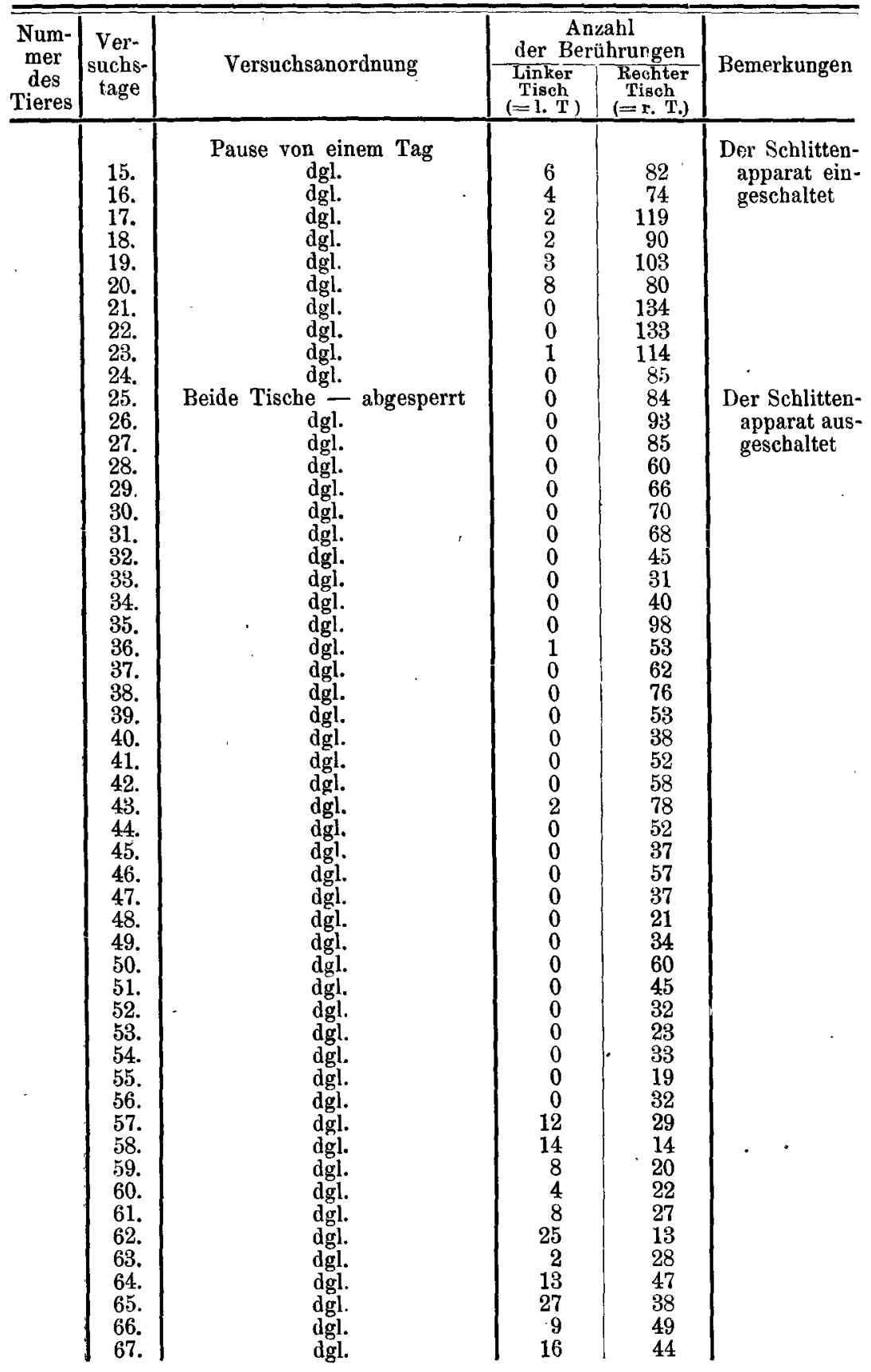


Beiträge zur Lehre von der. Entstehung neuer Gewohnheiten bei den Tieren. 133 Fortsetzung der Tabelle.

\begin{tabular}{|c|c|c|c|c|c|}
\hline \multirow[t]{2}{*}{$\begin{array}{c}\text { Num- } \\
\text { mer } \\
\text { des } \\
\text { Tieres }\end{array}$} & $\begin{array}{c}\text { Ver- } \\
\text { suchs- } \\
\text { tage }\end{array}$ & Versuchsanordnung & $\begin{array}{c}A \\
\operatorname{der} B e \\
\text { Linker } \\
\text { Tisch } \\
(\approx 1 . T .) \\
\end{array}$ & $\begin{array}{l}\text { ahl } \\
\text { hrungen } \\
\text { Rechter } \\
\text { Tisch } \\
(=x . \text { T.) }\end{array}$ & Bemerkungen \\
\hline & $\begin{array}{l}68 . \\
69 . \\
70 . \\
71 . \\
72 . \\
73 .\end{array}$ & $\begin{array}{l}\text { Beide Tische _- abgesperrt } \\
\text { dgl. } \\
\text { dgl. } \\
\text { dgl. } \\
\text { dgl. } \\
\text { dgl. }\end{array}$ & $\begin{array}{l}15 \\
23 \\
22 \\
12 \\
18 \\
10\end{array}$ & $\begin{array}{l}26 \\
15 \\
30 \\
12 \\
33 \\
19\end{array}$ & \\
\hline $\begin{array}{l}2 . \\
q\end{array}$ & $\begin{array}{l}1 . \\
2 . \\
3 . \\
4 . \\
5 . \\
6 . \\
7 . \\
8 . \\
9 .\end{array}$ & $\begin{array}{l}\text { Beide Tische - abgesperrt } \\
\text { dgl. } \\
\text { Der rechte Tisch - offen; der } \\
\text { linke Tisch - abgesperrt } \\
\text { dgl. } \\
\text { dgl. } \\
\text { dgl. } \\
\text { dgl. } \\
\text { Beide Tische - abgesperrt } \\
\text { dgl. }\end{array}$ & $\begin{array}{r}69 \\
27 \\
93 \\
120 \\
120 \\
122 \\
131 \\
210 \\
45\end{array}$ & $\begin{array}{r}78 \\
67 \\
2 \\
0 \\
1 \\
0 \\
0 \\
21 \\
77\end{array}$ & \\
\hline $\begin{array}{l}3 . \\
d\end{array}$ & $\begin{array}{l}1 . \\
2 . \\
3 . \\
4 . \\
5 . \\
6 . \\
7 . \\
8 . \\
9 . \\
10 . \\
11 . \\
12 . \\
13 . \\
14 . \\
15 . \\
16 . \\
17 . \\
18 . \\
19 . \\
20 .\end{array}$ & $\begin{array}{l}\text { Beide Tische - abgesperrt } \\
\text { Der rechte Tisch - offen; der } \\
\text { linke Tisch _ abgesperrt } \\
\text { dgl. } \\
\text { dgl. } \\
\text { dgl. } \\
\text { dgl. } \\
\text { dgl. } \\
\text { dgl. } \\
\text { Beide Tische - abgesperrt } \\
\text { dgl. } \\
\text { dgl. } \\
\text { dgl. } \\
\text { dgl. } \\
\text { dgl. } \\
\text { dgl. } \\
\text { dgl. } \\
\text { dgl. } \\
\text { dgl. } \\
\text { dgl. } \\
\text { dgl. }\end{array}$ & $\begin{array}{r}\infty \\
146 \\
74 \\
44 \\
157 \\
87 \\
91 \\
81 \\
71 \\
68 \\
64 \\
67 \\
83 \\
67 \\
41 \\
41 \\
14 \\
20 \\
32 \\
34 \\
\end{array}$ & $\begin{array}{r}\infty \\
8 \\
0 \\
0 \\
1 \\
0 \\
0 \\
0 \\
0 \\
0 \\
0 \\
0 \\
0 \\
0 \\
0 \\
33 \\
37 \\
37 \\
7 \\
28\end{array}$ & \\
\hline $\begin{array}{l}4 . \\
8\end{array}$ & $\begin{array}{l}1 . \\
2 . \\
3 . \\
4 . \\
5 . \\
6 . \\
7 . \\
8 . \\
9 . \\
10 . \\
11 . \\
12 .\end{array}$ & $\begin{array}{l}\text { Beide Tische - abgesperrt } \\
\text { Der rechte Tisch-abgesperrt; } \\
\text { der linke Tisch - offen } \\
\text { dgl. } \\
\text { dgl. } \\
\text { dgl. } \\
\text { dgl. } \\
\text { Beide Tische - abgesperrt } \\
\text { dgl. } \\
\text { dgl. } \\
\text { dgl. } \\
\text { dgl. } \\
\text { dgl. }\end{array}$ & $\begin{array}{r}66 \\
3 \\
0 \\
0 \\
0 \\
0 \\
0 \\
0 \\
0 \\
24 \\
45 \\
40\end{array}$ & $\begin{array}{l}36 \\
20 \\
43 \\
63 \\
52 \\
51 \\
67 \\
54 \\
39 \\
39 \\
36 \\
32\end{array}$ & \\
\hline
\end{tabular}


Noch übersichtlicher zeigt die graphische Darstellung den Versuchsverlauf (Abb. 4).

Diese Kurven schildern lückenlos das Verhalten der Versuchstiere während ihres kontinuierlichen, viele Tage und Nächte währenden Aufenthaltes im Apparate.

1.

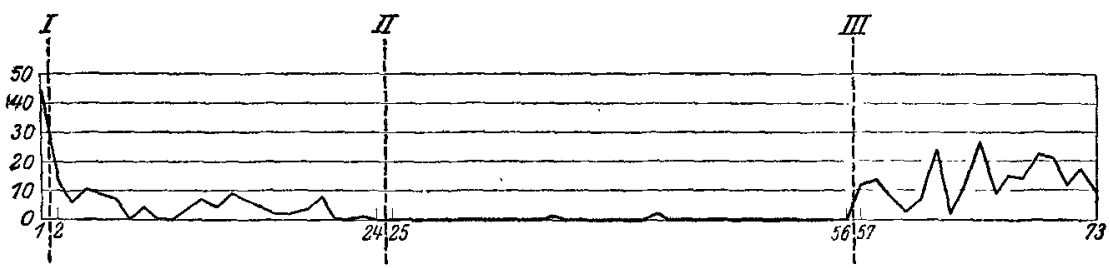

2.

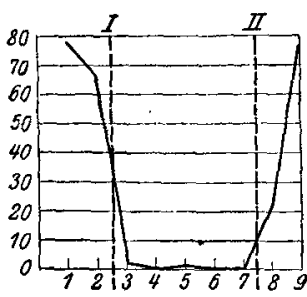

3.

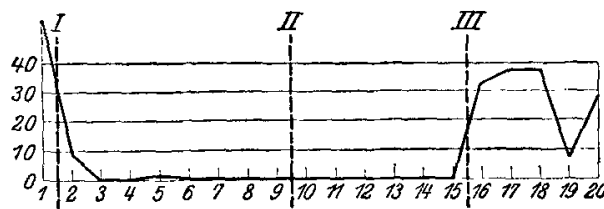

4.

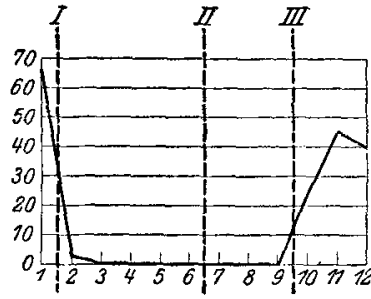

Abb. 4. Die Abbildungen $1-4$ beziehen sich auf die korrespondierenden Tiere. In sämtlichen Abbildungen trennen ab die gestrichelte Linie $I$ den Kontrollversuchstag von dem darauffolgenden Lernversuchstage, die gestrichelte Linie II die Lernversuchstage von den Versuchstagen, während welcher die erworbene Gewohnheit, einen bestimmten Tisch zu vermeiden, fortdauerte, die gestrichelte Linie III die zuletzt genaunten Versuchstage von den darauffolgenden Tagen, während welcher die erworbene Gewohnheit wiederum verklang. Auf den Abszissen sind die Versuchstage, auf den Ordinaten die Anzahl der Berührung des Tisches, den die Maus zu vermeiden erlernen sollte, eingetragen.

Das allgemeine Resultat dieser Versuche ergab zunächst, dass die Mäuse, den zureichenden Antrieb (elektrischer Schlag!) voraus- 
Beiträge zur Lehre von der Entstehung neuer Gewohnheiten bei den Tieren. 135

gesetzt, bereits nach einigen wenigen Erfahrungen während der ersten 24 Stunden lernen, den offenen Tisch zu vermeiden ${ }^{1}$ ).

Nach einigen Tagen der fehlerlosen Ausübung der erlernten Handlung (4-13 Tage) verklang die erworbene Gewohnheit ebenfalls raseh und unmittelbar.

- Im Falle der Maus 1, in dem der Lernvorgang infolge ursprünglich nicht zureichenden Antriebes ganz ausserordentlich lange währte, erstreckte sich auch das Fortbestehen der erworbenen Gewohnheit über eine ganz beträchtliche Reihe von Tagen (36 Tage). Der Vorgang des Verklingens der erworbenen Gewohnheit wich in nichts von den anderen Mäusen ab.

Bereits anlässlich ähnlicher Versuche an Kanarienvögeln habe ich die Vermutung ansgesprochen, dass der Verklingensvorgang einer erworbenen Gewohnheit sich zweierlei gestalten könnte. In einem Falle, den man als einen allmählichen Verklingensvorgang bezeichnen könnte, würde das Tier während der Verklingensperiode vereinzelte und wiederholte Versuche machen, die neue Gewohnheit zu üherwinden. Sollte dieser Fall wirklich zur Beobachtung gelangen, so müsste der Verklingensvorgang dem umgekehrt verlaufenden Lernvorgang ähneln.

In anderem Falle, der als ein plötzlicher Verklingensvorgang anzusehen wäre, würde sich die Verklingensperiode auf eine plötzliche und definitive Überwindung der erworbenen Gewohnheit reduzieren ${ }^{2}$ ).

Sowohl Kanarienvögel wie auch weisse Mäuse verhielten sich derart, dass die letztere Voraussetzung sich der Wirklichkeit anznnähern scheint.

Ein Umstand wäre hier noch erwähnenswert. Während einer ganzen Versuchsserie mit einer Maus blieb stets ein Tisch ununter-

1) Dicses Ergelnis bestätigte die Resultate meiner früheren Versuche an weissen Mäussen (1. c.). In der diesbezüglichen früheren Untersuchung legte ich ein anderes Prinzip („Mäusetage ${ }^{\text {) }}$ meiner Analyse des Lernvorganges zugrunde, da ich damals den biologisch richtigen Lernvorgang erschliessen wollte. In der vorliegenden Arbeit, in der das Augenmerk auf das Verklingen der erlernten Handlung gerichtet war, begnügte ich mich mit einem einfacheren Verfahren („Anzahl der Berührungen"), das ich für genügend für meinen Zweck hielt. Nichtsdestoweniger waren die Ergebnisse beider Versuchsreihen übereinstimmend.

2) Vgl. „Abhandlungen zum Aufbau der Lehre von den Handlungen der Tiere" (Pflüger's Arch. Bd. 170 S. 194-195. 1918). 
brochen abgesperrt, so dass eigentlich das Tier eine doppelte Erfahrung machen masste, und zwar einen bestimmten Tisch aufzusuchen, falls dasselbe Hunger hatte, und einen anderen Tisch stets zu vermeiden.

Infolgedessen wäre es denkbar, dass während der Verklingensperiode sich ein Widerstreit zwischen zwei Erfahrungen einstellen könnte. Denn zu der früheren Erfahrung, zu dem bestimmten Futtertisch zu laufen, gesellt sich nun die neue Erfahrung, auch zu dem anderen, bisher unzugänglichen Futtertisch einen bequemen Zugang zu finden. Es könnte sein, dass die Mäuse sich ähnlich verhalten würden, wie sich die Hühner unter ähnlichen Bedingungen benommen haben. Für die letzteren Tiere haben nämlich $\mathrm{Katz}$ und $\mathrm{Révész}$ folgende Regelmässigkeit feststellen können:

"Wird eine oft gemachte und fest eingeprägte Erfahrung durch die entgegengesetzte auf geringerer Erlernungszahl beruhende Erfahrung in ihrem Einfluss gehemmt, so verhält es sich eine Zeitlang im Sinne dieser letzteren Erfahrung. Nach wenigen Stunden jedoch ist ilas Stärkeverhältnis der jüngeren Frfahrung zur älteren ein solches geworden, dass das Huhn sich wieder im Sinne der älteren verhält" $\left.{ }^{\prime \prime}\right)$.

Diese Regelmässigkeit zeigten meine Mäuse nicht; sie verhielten sich derart, als ob kein Widerstreit der Erfahrungen sich geltend gemacht hätte.

Die Tiere liefen von dem Momente des Verklingens ungefähr ebenso oft zu dem einen wie zu dem anderen Futtertische, so dass keine ausgesprochene Bevorzugung eines der Tische zu beobachten war.

Zum Schluss möchte ich noch bemerken, dass die Mäuse 1 und 2 zwischen 6 und $7 \mathrm{Uhr}$ früh, die Mäuse 3 und 4 zwischen 7 und $10 \mathrm{Uhr}$ abends den früher offenen, jetzt abgesperrten Tisch zum erstenmal während der Verklingensperiode berührt haben.

Da die weissen Mäuse die intensivste Beweglichkeit während der Abend- bzw. Nachtstunden äussern, könnte man glauben, dass auch das Verklingen der früheren Erfahrung sich auch während dieser Zeit zum erstenmal geltend machen würde.

Die Tatsachen bestätigten nicht diese Vermutung.

1) D. Katz und G. Révész, Experimentell-psychologische Untersuchungen mit Hühnern (Sonderabdr. a. Zeitschr. f. Psychol. Bd. 50 S. 108. 1908). 


\section{Ein Versuch über die Fähigkeit der Tiere, Erfahrungen $z \mathfrak{u}$ verallgemeinern.}

Die Frage, ob ein Tier eine früher gemachte und auf einen bestimmten Einzelfall bezogene Erfahrung verallgemeinern kann, ist sehr schwer zu entscheiden.

Ein methodisches Verfahren, das hier guten Dienst leisten könnte, schien mir darin zu bestehen, dass man ein Tier in eine bestimmte, stets gleichbleibende Umgebung versetzen würde, in der eine feste Verknüpfung zwischen einem bestimmten Element dieser Umgebung und der einzuschlagenden Bewegungsrichtung im Versuchstiere entstehen müsste. Daraufhin würde man das Tier in eine ihn bisher fremde Umgebung bringen, in der bloss jenes Element, mit dem das Tier als mit dem sensorischen Bestandteil einer früher fest ausgebildeten sensorisch-motorischen Verknüpfung vertraut war, von der alten Umgebung her übernommen würde. Wenn nun dieses Element auf das Tier richtungbestimmend wirken sollte, könnte man vermuten, dass das Tier die frühere Erfahruug auch unter den neuen Bedingungen gelten liesse; man könnte in diesem Falle mit Recht von der Verallgemeinerung der Erfahrung reden. Unter Zugr undelegung dieser Voraussetzung wurden nun folgende Versuche an weissen Ratten ausgeführt:

Zunächst wurden die Tiere in einem Labyrinth, dessen Grundriss die Abb. 55 bringt, untersucht (Abb. 5).

Die Scheidewände des Labyrinthes $O$ bzw. $u$ (Abb. 53) stellten die Brettchen $o$ bzw. $u$ (Abb. 54) dar, so dass die Ratte, um weiterzukommen, entweder unterkriechen (u) oder überkriechen (o) musste.

Nachdem es sich herausgestellt hatte, dass die Tiere keine der beiden möglichen Bewegungsformen bevorzugten, wurden die Tiere (zweimal täglich) im Apparate, dessen Grundriss die Abb. 51 bringt, untersucht ${ }^{1}$ ).

Dieser schwarz angestrichene Apparat bestand aus einem Kasten $x y$ ohne Boden und ohne Deckel; dessen Dimensionen waren: Höhe $35 \mathrm{~cm}$; Länge $43 \mathrm{~cm}$; die grösste Breite $34 \mathrm{~cm}$. Durch die Scheidewände $d c$ und $a b$ war das Innere in drei Abteilungen geteilt; in der

1) Ich brauche wohl kaum besonders zu erwähnen, dass der Boden und die Fenster nach jedem Versuch mit Seifenwasser bzw. Äther gründlich abgewaschen wurden. 
Wand $w x$ war je ein Fenster, das dem Tier ermöglichte, den Apparat zu verlassen und zu dem dicht nebenan angebrachten Wóhnkäfig zu gelangen. Jedes der beiden letzteren Fenster konnte nach Belieben durch eine Tür aus Drahtnetz abgesperrt werden; gleich hier sei erwähnt, dass bei den letzten zehn Versuchen beide Fenster zur Kontrolle offengelassen wurden.

In der Wand $a b$ wurden zwei Fenster $(5 \times 6 \mathrm{~cm})$ derart ausgeschnitten, wie dies in der Abb. 52 dargestellt ist. Die bei $z$ ein-

$I$.

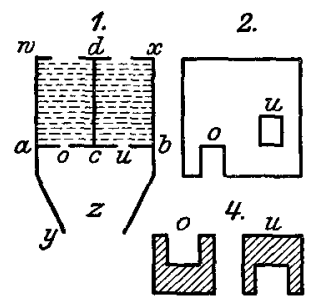

3.

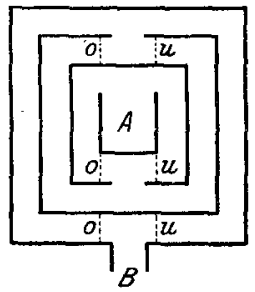

$I I$.

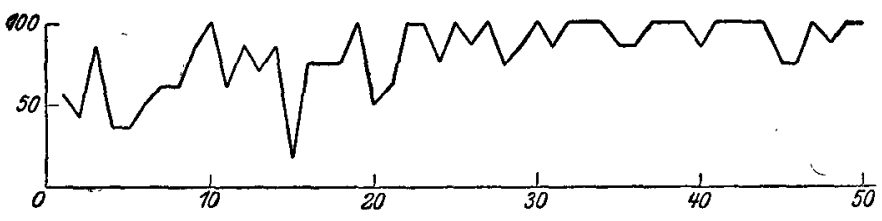

Abb. 5 .

I. 1: Grundriss des Apparates (die gestrichelten Linien in $a b H w$ deuten den "elektrischen Boden" an); 2: das Brett, das in der Abb. 1 die Wand al ausmachte; 3: das Labyrinth; 4: zwei Arten von ausgeschnittenen Brettchen, die die Scheidewände des Labyrinthes bei $o$ bzw. $u$ ausmachten.

II. Lernkurve der Ratten. (Durchschnitt von sämtlichen untersuchten Tieren.) Auf der Abszisse sind Versuchstage, auf der Ordinate die Ánzahl der richtigen Fälle in Prozent eingetragen.

gebrachte Ratte musste nun, um in die eine der Abteilungen $a d$ bzw. $b d$ zu gelangen, entweder unterkriechen ( $u$ Abb. 52 ) oder überkriechen (o). Durch das Umdrehen der Scheidewand $a b$ um $180^{\circ}$ konuten beide Fenster gewechselt werden ${ }^{2}$ ).

Die Tiere, die nur nach dem Versuche Futter erhielten, mussten nun erlernen, wenn sie in die Abteilung $z$ (Abb. 5 1) gesetzt wurden,

1) Die Reihenfolge, in der das wirksame Fenster wechselte, war die folgende: $\operatorname{lrlr} \ln r \ln \ln 11 \mathrm{r} \operatorname{lr}(\mathrm{r}$ ist rechts, 1 ist links). 
Beiträge zur Lehre von der Entstehung neuer Gewohnheiten bei den Tieren. 139

stets das höher gelegene Fenster (überkriechen) zu erwählen, falls sie zum Wohnkäfig gelangen wollten.

Wenn das Tier das falsche Fenster bevorzugte, fand dasselbe nicht nur den Ausgang versperrt, sondern erhielt ausserdem noch einen elektrischen Schlag. Dies letztere ermöglichte ein in den Raum $a b w x$ (Abb. 51) angebrachter "elektrischer Boden“.

Diese Versuchsanordnung wurde deshalb gewählt, weil die Ratten, als in der Natur unterirdisch lebende Tiere, sich durch alle möglichen Arten von Löchern durchzwängen müssen. Das Unterscheiden zwischen Unter- und Überkriechen musste also den natürlichen Lebensbedingungen der Tiere gut angepasst und deshalb relativ leicht zu erlernen sein.

Dass diese Voraussetzung richtig war, zeigten die Versuchsergebnisse, die in der nächstfolgenden Tabelle und in der Kurve (Abb. 5, II) dargestellt sind.

\begin{tabular}{|c|c|c|c|c|c|c|c|c|c|}
\hline $\begin{array}{l}\text { Nummer } \\
\text { des } \\
\text { Versuches }\end{array}$ & $\begin{array}{l}1 . \\
7\end{array}$ & $\begin{array}{l}2 . \\
q\end{array}$ & $\begin{array}{l}3 . \\
\delta\end{array}$ & $\begin{array}{l}4 . \\
\delta\end{array}$ & $\begin{array}{l}5 . \\
q\end{array}$ & $\begin{array}{l}6 . \\
\text { ठ }\end{array}$ & 7. & $\begin{array}{l}8 . \\
7\end{array}$ & $\begin{array}{l}\text { Richtige Falle } \\
\text { in Prozent } \\
\text { (Durchschnitt } \\
\text { ron } 1-8 \text { ) }\end{array}$ \\
\hline 1. & $?$ & - & + & + & - & + & - & + & 57 \\
\hline 2. & $?$ & - & - & - & + & 1 & + & + & 43 \\
\hline 3. & + & $\ldots$ & $t$ & + & $?$ & + & + & + & $86^{\circ}$ \\
\hline 4. & - & - & + & + & $\ldots$ & - & - & + & 37,5 \\
\hline 5. & - & + & + & + & 一 & + & - & + & 37,5 \\
\hline 6. & - & + & + & - & - & - & + & + & 50 \\
\hline 7. & - & + & + & + & + & + & 一 & - & 62,5 \\
\hline 8. & + & - & + & + & - & - & + & + & 62,5 \\
\hline 9. & + & + & + & + & + & + & - & + & 87,5 \\
\hline 10. & + & + & + & + & + & + & + & + & 100 \\
\hline 11. & - & - & + & + & $\therefore$ & + & $\therefore$ & + & 50 \\
\hline 12. & + & - & + & + & + & + & $?$ & + & 86 \\
\hline 13. & - & + & + & + & - & + & $?$ & + & 71 \\
\hline 14. & + & + & + & + & + & - & + & + & $87,-5$ \\
\hline 15. & - & + & - & - & - & + & $?$ & - & 18 \\
\hline 16. & + & 一 & + & + & + & - & + & + & 75 \\
\hline 17. & + & - & + & + & - & + & + & + & 75 \\
\hline 18. & - & + & + & + & + & - & + & + & 75 \\
\hline 19. & + & + & $t$ & + & + & + & + & + & 100 \\
\hline 20 & - & + & - & + & - & + & - & + & 50 \\
\hline 21. & - & - & + & + & + & + & + & - & 62,5 \\
\hline 22. & + & + & + & + & + & + & + & + & 100 \\
\hline 23. & + & + & + & + & + & + & + & + & 100 \\
\hline 24. & + & - & + & + & + & + & + & - & 75 \\
\hline 25. & + & + & + & + & + & + & + & + & 100 \\
\hline 26. & + & - & + & + & + & + & + & + & 87,5 \\
\hline 27. & + & + & + & + & + & + & + & + & $100^{\circ}$ \\
\hline 28. & + & $t$ & + & - & + & + & - & + & 75 \\
\hline 29. & -- & + & + & + & + & + & + & + & 87,5 \\
\hline 30. & + & + & + & + & + & + & + & + & 100 \\
\hline
\end{tabular}


Fortsetzung der Tabelle.

\begin{tabular}{|c|c|c|c|c|c|c|c|c|c|}
\hline $\begin{array}{l}\text { Nummer } \\
\text { des } \\
\text { Verstches }\end{array}$ & $\begin{array}{l}1 . \\
\text { q }\end{array}$ & $\begin{array}{l}2 . \\
\text { 우 }\end{array}$ & $\begin{array}{l}3 . \\
\delta\end{array}$ & $\begin{array}{l}4 . \\
\delta\end{array}$ & $\begin{array}{l}5 . \\
\text { 오. }\end{array}$ & $\begin{array}{l}6 . \\
\delta\end{array}$ & 7. & $\begin{array}{l}8 . \\
\text { 우 }\end{array}$ & $\begin{array}{l}\text { Richtige Fälle } \\
\text { in Prozent } \\
\text { (Durchschnitt } \\
\text { von } 1-8 \text { ) }\end{array}$ \\
\hline $\begin{array}{c}31 . \\
32 . \\
33 . \\
34 . \\
35 . \\
36 . \\
37 . \\
38 . \\
39 . \\
40 . \\
41 . \\
42 . \\
43 . \\
44 . \\
45 . \\
46 . \\
47 . \\
48 . \\
49 . \\
50 .\end{array}$ & $\begin{array}{l}+ \\
+ \\
+ \\
+ \\
+ \\
+ \\
+ \\
+ \\
+ \\
+ \\
+ \\
+ \\
+ \\
+ \\
+ \\
+ \\
+ \\
+ \\
+ \\
+\end{array}$ & $\begin{array}{l}+ \\
+ \\
+ \\
+ \\
+ \\
+ \\
+ \\
+ \\
+ \\
+ \\
+ \\
+ \\
+ \\
+ \\
+ \\
+ \\
+ \\
+ \\
+ \\
+\end{array}$ & $\begin{array}{l}+ \\
+ \\
+ \\
+ \\
+ \\
+ \\
+ \\
+ \\
+ \\
+ \\
+ \\
+ \\
+ \\
+ \\
+ \\
+ \\
+ \\
+ \\
+ \\
+\end{array}$ & $\begin{array}{l}+ \\
+ \\
+ \\
+ \\
+ \\
+ \\
+ \\
+ \\
+ \\
+ \\
+ \\
+ \\
+ \\
+ \\
+ \\
+ \\
+ \\
+ \\
+ \\
+\end{array}$ & $\begin{array}{l}+ \\
+ \\
+ \\
+ \\
+ \\
+ \\
+ \\
+ \\
+ \\
+ \\
+ \\
+ \\
+ \\
+ \\
+ \\
+ \\
+ \\
+ \\
+ \\
+\end{array}$ & $\begin{array}{l}+ \\
+ \\
+ \\
+ \\
+ \\
+ \\
+ \\
+ \\
+ \\
+ \\
+ \\
+ \\
+ \\
+ \\
+ \\
+ \\
+ \\
+ \\
+ \\
+\end{array}$ & $\begin{array}{l}+ \\
+ \\
+ \\
+ \\
+ \\
+ \\
+ \\
+ \\
+ \\
+ \\
+ \\
+ \\
+ \\
+ \\
+ \\
+ \\
+ \\
+ \\
+ \\
+\end{array}$ & $\begin{array}{l}+ \\
+ \\
+ \\
+ \\
+ \\
+ \\
+ \\
+ \\
+ \\
+ \\
+ \\
+ \\
+ \\
+ \\
+ \\
+ \\
+ \\
+ \\
+\end{array}$ & $\begin{array}{c}87,5 \\
100 \\
100 \\
100 \\
87,5 \\
87,5 \\
100 \\
100 \\
100 \\
87,5 \\
100 \\
100 \\
100 \\
100 \\
75 \\
75 \\
100 \\
87,5 \\
100 \\
100\end{array}$ \\
\hline
\end{tabular}

Die Tabelle und die Kurve zeigen, dass die Ratten bereits nach ca. 21 Versuchen gelernt batten, das obengelegene Fenster (überkriechen) zu benützen, um zum Wohnkäfig zu gelangen und dem elektrischen Schlag zu entgehen.

Nachdem diese Ergebnisse festgestellt waren (nach 50 Versuchen), wurden die Ratten neuerdings im bereits beschriebenen Labyrinth (Abb. 5 3) untersucht. Fs sollte festgestellt werden, ob die Tiere nun jene Labyrinthsgänge bevorzugen werden, die durch das Brettrhen $o$ (üherkriechen, Abb. 54) abgesperrt waren. Es schien mir nicht ratsam, mehr als zwei Versuche (und zwar in der gleichen Zeit und bei der gleichen Diät wie die früheren Lernversuche) auszuführen.

Das Ergebnis war in der Mehrzahl der Fälle negativ. Die Ratten zeigten sich im Labyrinth eingeschüchtert und wollten sich kaum bewegen.

Bloss die Ratten Nr. 6, 7, 8 bewegten sich im Labyrinth ganz frei und gelangten nach einer kurzen Zeit von $B \mathrm{zu}$ dem im Labyrinthzentrum $(A, \mathrm{Abb} .53)$ aufgestellten Käfig. Diese Ratten benützten allerdings in $80 \%$ die durch das Brettchen $o$ (überkriechen) abgesperrten Gänge, so dass eine Verallgemeinerung der früher gemachten Erfahrung in diesen Fällen möglicherweise stattgefunden hat. 
Beiträge zur Lehre von der Entstehung neuer Gewohnheiten bei den Tieren. 141

Trotz dieses letzteren Ergebnisses scheint es mir indessen vorsichtiger zu sein, daran zu glauben, dass die Ratten die Umgebung als das Ganze rezipieren würden und ausserstande wären, dieselbe in ibre Bestandteile zú zergliedern und bloss einen der letzteren in einem. neuen Zusammenhange zu reproduzieren.

Dieser Ansicht sind auch andere Forscher auf dem Gebiete der Tierpsychologie.

\section{Die Abhängigkeit der Lerngeschwindigkeit von der Antriebsstärke.}

Die vorliegende Untersuchung ging dem Problem nach, ob es eine Abhängigkeit zwischen der Lerngeschwindigkeit und der Antriebsstärke gibt.

Un dieser Frage näherzukommen, habe ich die Versuche an weissen Mäusen mit der Labyrinthmethode ausgeführt. Die Versuchstiere wurden in zwei Serien verteilt, von denen die eine das ganz einfache Labyrinth unter der Einwirkung von einem stärkeren Antrieb, die andere unter der Einwirkung von einem schwächeren Antrieb erlernen sollte.

Um einen starken Antrieb zur Ausführung der Handlung gelten zu lassen, liess ich die Mäuse der ersten Serie das Labyrinth von der Mitte bis zum Ausgang auf einem erhitzten Boden durchlaufen (die Methode des "erhitzten Bodens" 1 ). Einen schwachen Antrieb zur Ausführung der Handlung liess ich bei den Tieren der zweiten Serie dadurch entstehen, dass die Tiere den Weg im Labyrinth auf einem feuchten Boden zurücklegen mussten (die Methode des "feuchten Bodens").

Die Versuchsanordnung bei der Methode des "erhitzten Bodens" war die folgende (Abb. 6, I).

Auf einen allseits geschlossenen Kasten aus Zinkblech $(30 \mathrm{qcm}$, $5 \mathrm{~cm}$ hoch) $(A B)$, der durch das Rohr $x$ mit Wasser vollgefüllt war,

1) Die Methode des "erhitzten Bodens" ruft eine historische Reminiszenz wach, die Mangin in seinem Buche über "Mensch und Tier" bringt. Im Jahre 1810 in Smorgon (Lithauen) und in Klewan (Wolhynien) waren zwei Bärenakademien ... Man führte die Bären mit in Lappen umwickelten Hinterpfoten in die ad hoc eingerichteten Öfen hinein; die Wärme zwang die Tiere, die Vorderpfoten aufzuheben, auf diese Weise lernten sie Tanz und viel Bewegung (zit. nach Breh m)。 
war das Labyrinth $E F$ gestellt. Dicht an den Kasten, und zwar dem Labyrinthausgang gegenüber, war der Käfig $G H$ auf einem Brett derart angebracht, dass die Maus aus dem Labyrinthausgang direkt in den Käfig-hineinschlüpfen konnte.

Empirisch konnte man feststellen, dass es genügte, die Tempera-

I.

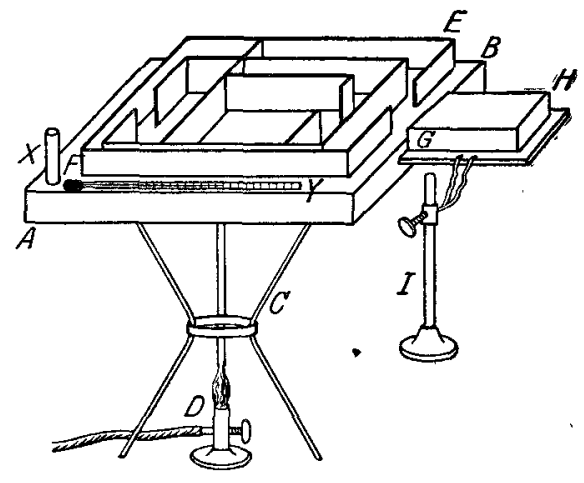

III.

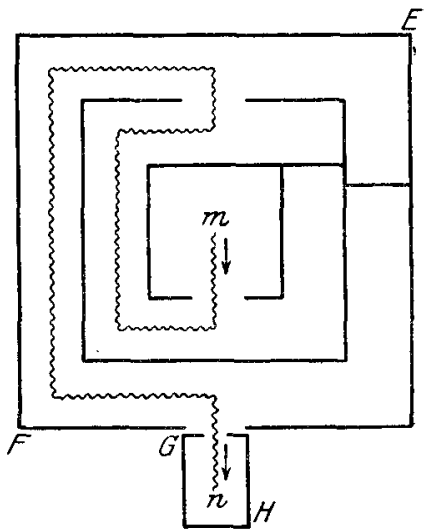

II.

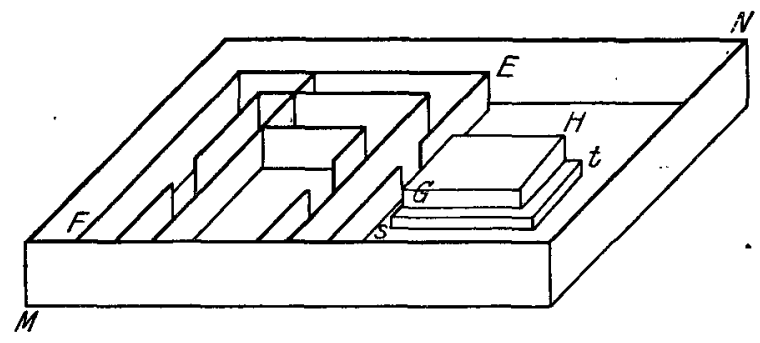

Abb. 6.

I. Methode des erhitzten Bodens. $A B$ : heizbarer Boden $(x:$ Rohr $; y$ : Thermometer); $E F$ : Labyrinth; $G H$ : Kätig.

II. Methode des feuchten Bodens. MN: Wanne mit einer $5 \mathrm{~mm}$ tiefen Wasserschicht; $E F$ : Labyrinth; $G H$ : Käfig.

1II. Grundriss des Labyrinthes $E F$ und des Käfigs $G H$; die gewellte Linie $m n$ zeigt den kürzesten Weg zwischen Labyrinthmitte $m$ und dem Käfiginnern $n$.

tur der Kastenoberfläche so weit zu erhöhen, dass ein darauf gelegtes Thermometer die Temperatur von $41-43^{\circ} \mathrm{C}$. zeigte.

Bei dieser Temperatur nahmen die Mäuse, wie mir dies die uumittelbare und die mehrere Tage nach dem Versuche fortgesetzte Beobachtung zeigte, keinen Schaden. Die erste, allerdings nur schwache Reaktion (Belecken der Sohlen) stellte sich bereits bei einer Tem- 
peratur von $37^{\circ} \mathrm{C}$. ein. Bei der Temperatur von $41-43^{\circ} \mathrm{C}$. gestaltete sich das Verhalten der Tiere folgendermaassen: Bei dem Ansetzen auf dem erhitzten Boden blieben die Tiere auf dem gleichen Fleck sitzen und beleckten oft und stark die Sohlen; daraufhin liefen sie eine kurze Strecke fort und wiederholten die gleiche Handlung; erst allmählich erlernten die Tiere, das Belecken vollständig bzw. nahezu vollständig zu unterlassen und direkt zum Labyrinthausgang zu laufen.

Ich habe den Eindruck gewonnen, dass die Tiere zu Anbeginn der Versuche die Örtlichkeit der Reizquelle in die Fusssohlen verlegen und erst erlernen müssen, dieselbe als an den Labyrinthboden gebunden anzuseben und infolgedessen das Labyrinth auf dem kürzesten Wege zu verlassen.

Die Methode des "feuchten Bodens" bestand darin, dass das Labyrinth (Abb. 6, $I I, F E$ ) in einer geräumigen, aber flachen Porzellanwanne $(M N)$ angebracht war. Der Wannenboden war mit einer Wassersehicht von $5 \mathrm{~mm}$ Tiefe und von $18^{\circ} \mathrm{C}$. bedeckt. Der bereits erwähnte Käfig $G H$ war dicht an den Labyrinthausgang anf einem Brett $s t$, das über die Wasserlinie herausragte, angebracht.

Das Verhalten der Maus auf dem feuchten Boden war von jenem auf dem erhitzten Boden ganz abweichend: Wenn die Maus in dem letzteren Falle, sobald sie das Belecken zu unterlassen erlernte und einmal den Käfig gefunden hatte, bei den nächsten Versuchen das Labyrinth auf dem kürzesten Wege und in der Regel sobald wie möglich zu verlassen trachtete, so verhielt sich im Gegenteil die,Maus auf dem feuchten Borlen viel ruhiger. Dieselbe untersuchte sehr genau den Boden und die Labyrinthwände und beeilte sich nicht, auf dem kürzesten Wege den trockenen Käfig zu erreichen; es ist auch öfters vorgekommen, dass die Maus im Verlaufe des Lernvorganges den Labyrinthausgang bereits auf dem kürzesten Wege erreichte; daraufhin aber machte sie, statt sich in den Käfig zu verkriechen, kehrt und lief das Labyrinth nochmals durch.

Wie dieses Verhalten vermuten lässt, erwies sich die anfangs gemachte Voraussetzung, dass der erhitzte Boden einen stärkeren Antrieb als der feuchte erzeugen müsse, als vollkommen berechtigt.

Das Labyrinth, mit dem diese Versuche ausgeführt waren, hat einen ganz einfachen Grundriss (Abb. 6, III); dasselbe war aus schwarz angestrichenem Holz verfertigt, $28 \mathrm{qcm}$ im grössten Umfang und $4 \mathrm{~cm}$ 
hoch; von oben war das Labyrinth mit einem feinen Drahtnetz bespannt. Bloss der Zentralraum $m$ blieb offen und konute nach Belieben mit einer Glasscheibe bedeckt werden.

Der Verlauf einer einzelnen Versuchsserie mit einer Maus war der folgende: Nachdem das Tier in den Zentralraum des Labyrinthes $m$ (Abb. 6, III) getan worden war, wurde die Öffnung mit einer Glasscheibe verdeckt. Sobald die Maus das Käfiginnere $n$ betreten hatte, wurde der Käfigeingang mit einer bereitliegenden Glasscheibe versperrt; darauf hin wurde der Boren mit Seifenwasser abgewaschen und nach einer Pause von 5 Minuten die Maus wiederum ins Labyrinth getan usf. Nachdem die Maus in drei aufeinanderfolgenden Versuchen das Labyrinth auf dem kürzesten Wege zuruckgelegt hatte, nahm der Versuch ein Ende.

Für die Auswertung der Resultate schien mir von ausschlaggebender Bedeutung der jedesmal zurückgelegte Weg zu sein. Um mir von dem letzteren Rechenschaft geben zu können, legte ich der Beurteilung der Versuchsresultate die Anzahl der Fehler bei jedem Versuche zugrunde. Da die Fehler sich auf das Abweichen vóm kürzesten Wege (Betreten eines falschen Ganges bzw. Umkehren in einem richtigen Gange) bezogen, gaben dieselben einen Begriff vom jedesmal zurihckgelegten Wege.

Je fünf erwachsene weisse Mäuse wurden in jeder Serie untersucht; Nr. 1, 3, 4, 9 waren Weibchen, 2, 5, 6, 7, 8, 10 Männchen.

Die genauen Versuchsergebnisse sind in der nächstfolgenden Tabelle niedergelegt.

\begin{tabular}{|c|c|c|c|c|}
\hline Versuchsanordnung & $\begin{array}{c}\text { Nummer } \\
\text { des } \\
\text { Tieres }\end{array}$ & $\begin{array}{l}\text { Nummer } \\
\text { des } \\
\text { Versuches }\end{array}$ & Fehler & Zeit \\
\hline \multirow{3}{*}{$\begin{array}{l}\text { "Erhitzter Boden" bis auf } \\
41-43^{\circ} \text { C. (Erwachsene } \\
\text { Tiere.) }\end{array}$} & 1 & $\begin{array}{l}1 \\
2 \\
3 \\
4 \\
5\end{array}$ & $\begin{array}{l}7 \\
1 \\
0 \\
0 \\
0\end{array}$ & $\begin{array}{r}315 \\
108 \\
12 \\
3 \\
3\end{array}$ \\
\hline & 2 & $\begin{array}{l}1 \\
2 \\
3 \\
4 \\
5\end{array}$ & $\begin{array}{r}22 \\
4 \\
0 \\
0 \\
0\end{array}$ & $\begin{array}{r}635 \\
193 \\
74 \\
49 \\
19\end{array}$ \\
\hline & 3 & $\begin{array}{l}1 \\
2 \\
3\end{array}$ & $\begin{array}{r}11 \\
1 \\
2\end{array}$ & $\begin{array}{c}? \\
120 \\
199\end{array}$ \\
\hline
\end{tabular}


Beiträge zur Lehre von der Entstehung ṇeuer Gewohnheiten bei den Tieren. 145 Fortsetzung der Tabelle.

\begin{tabular}{|c|c|c|c|c|}
\hline Versuchsanordnung & $\begin{array}{l}\text { Nummer } \\
\text { des } \\
\text { Tieres }\end{array}$ & $\begin{array}{l}\text { Nummer } \\
\text { des } \\
\text { Versuches }\end{array}$ & Fehler & Zeit \\
\hline \multirow[t]{3}{*}{$\begin{array}{l}\text { "Erhitzter Boden" bis auf } \\
41-43^{\circ} \text { C. (Erwachsene } \\
\text { Tiere.) }\end{array}$} & 3 & $\begin{array}{l}4 \\
5 \\
6 \\
7 \\
8\end{array}$ & $\begin{array}{l}1 \\
2 \\
0 \\
0 \\
0\end{array}$ & $\begin{array}{r}97 \\
92 \\
199 \\
171 \\
35\end{array}$ \\
\hline & 4 & $\begin{array}{l}1 \\
2 \\
3 \\
4\end{array}$ & $\begin{array}{l}1 \\
0 \\
0 \\
0\end{array}$ & $\begin{array}{c}300 \\
125 \\
? \\
8\end{array}$ \\
\hline & 5 & $\begin{array}{l}\mathbf{1} \\
2 \\
3 \\
4 \\
5 \\
6 \\
7 \\
8\end{array}$ & $\begin{array}{r}5 \\
10 \\
3 \\
1 \\
1 \\
0 \\
0 \\
0\end{array}$ & $\begin{array}{c}207 \\
158 \\
73 \\
28 \\
? \\
14 \\
3 \\
4\end{array}$ \\
\hline \multirow[t]{3}{*}{$\begin{array}{l}\text { "Feuchter Boden“. } \\
\text { wachsene Tiere.) }\end{array}$} & 6 & $\begin{array}{r}1 \\
2 \\
3 \\
4 \\
5 \\
6 \\
7 \\
8 \\
9 \\
10 \\
11 \\
12 \\
13 \\
14\end{array}$ & $\begin{array}{l}1 \\
1 \\
1 \\
1 \\
5 \\
1 \\
2 \\
5 \\
1 \\
0 \\
1 \\
0 \\
0 \\
0\end{array}$ & $\begin{array}{r}149 \\
62 \\
40 \\
42 \\
? \\
30 \\
127 \\
? \\
89 \\
111 \\
85 \\
115 \\
21 \\
182\end{array}$ \\
\hline & 7 & $\begin{array}{l}1 \\
2 \\
3 \\
4 \\
5 \\
6 \\
7\end{array}$ & $\begin{array}{l}7 \\
1 \\
1 \\
1 \\
0 \\
0 \\
0\end{array}$ & $\begin{array}{r}229 \\
72 \\
28 \\
47 \\
27 \\
17 \\
?\end{array}$ \\
\hline & 8 & $\begin{array}{r}1 \\
2 \\
3 \\
4 \\
5 \\
6 \\
7 \\
8 \\
9 \\
10\end{array}$ & $\begin{array}{l}3 \\
4 \\
6 \\
2 \\
6 \\
2 \\
4 \\
0 \\
0 \\
0\end{array}$ & $\begin{array}{r}347 \\
158 \\
262 \\
105 \\
270 \\
130 \\
321 \\
241 \\
58 \\
40\end{array}$ \\
\hline
\end{tabular}


J. S. Szymanski:

Fortsetzung der Tabelle.

\begin{tabular}{|c|c|c|c|c|}
\hline Versuchsanordnung & $\begin{array}{l}\text { Nummer } \\
\text { des } \\
\text { Tieres }\end{array}$ & $\begin{array}{l}\text { Nummer } \\
\text { des } \\
\text { Versuches }\end{array}$ & Fehler & Zeit \\
\hline $\begin{array}{l}\text { "Feuchter Boden". (Er- } \\
\text { wachsene Tiere.) }\end{array}$ & - & $\begin{array}{r}1 \\
2 \\
3 \\
4 \\
5 \\
6 \\
7 \\
8 \\
9 \\
10 \\
11 \\
\\
1 \\
2 \\
3 \\
4 \\
5 \\
6 \\
7 \\
8 \\
9 \\
10 \\
11 \\
12 \\
13 \\
14 \\
15 \\
16 \\
17 \\
18 \\
19 \\
20 \\
21 \\
22 \\
23 \\
24 \\
25 \\
30 \\
3\end{array}$ & 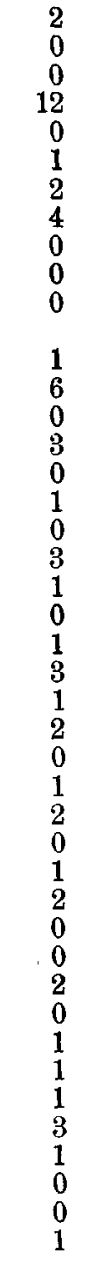 & $\begin{array}{r}85 \\
29 \\
27 \\
295 \\
31 \\
58 \\
173 \\
234 \\
58 \\
61 \\
29 \\
\\
110 \\
173 \\
32 \\
132 \\
26 \\
36 \\
31 \\
191 \\
210 \\
20 \\
73 \\
107 \\
58 \\
59 \\
87 \\
62 \\
101 \\
45 \\
? \\
134 \\
50 \\
? \\
61 \\
23 \\
41 \\
90 \\
43 \\
253 \\
40 \\
121 \\
27 \\
25\end{array}$ \\
\hline $\begin{array}{l}\text { "Erhitzter Boden" bis auf } \\
41-43^{\circ} \text { C. (Junge Tiere.) }\end{array}$ & 11 & $\begin{array}{l}1 \\
2 \\
3 \\
4 \\
5 \\
6 \\
7 \\
8\end{array}$ & $\begin{array}{r}15 \\
12 \\
3 \\
2 \\
2 \\
0 \\
0 \\
0\end{array}$ & $\begin{array}{l}- \\
- \\
- \\
-\end{array}$ \\
\hline
\end{tabular}


Beiträge zur Lehre von der Entstehung neuer Gewohrheiten bei den Tieren. 147

Fortsetzung der Tabelle.

\begin{tabular}{|c|c|c|c|c|}
\hline Versuchsanordnung & $\begin{array}{c}\text { Nummer } \\
\text { des } \\
\text { Tieres }\end{array}$ & $\begin{array}{c}\text { Nummer } \\
\text { des } \\
\text { Versuches }\end{array}$ & Fehler & Zeit \\
\hline \multirow[t]{2}{*}{$\begin{array}{l}\text { "Erhitzter Boden" bis auf } \\
41-43^{0} \text { C. (Junge Tiere.) }\end{array}$} & 12 & $\begin{array}{l}1 \\
2 \\
3 \\
4 \\
5 \\
5 \\
6 \\
7 \\
8 \\
9\end{array}$ & $\begin{array}{r}10 \\
\mathbf{2} \\
4 \\
1 \\
2 \\
3 \\
0 \\
0 \\
0\end{array}$ & $\begin{array}{l}= \\
= \\
= \\
=\end{array}$ \\
\hline & 13 & $\begin{array}{r}1 \\
2 \\
3 \\
4 \\
5 \\
6 \\
7 \\
8 \\
9 \\
10 \\
11 \\
12 \\
13 \\
14 \\
15\end{array}$ & $\begin{array}{l}6 \\
4 \\
2 \\
1 \\
1 \\
2 \\
2 \\
6 \\
1 \\
2 \\
2 \\
1 \\
0 \\
0 \\
0\end{array}$ & $\begin{array}{l}- \\
= \\
= \\
= \\
= \\
= \\
= \\
= \\
= \\
=\end{array}$ \\
\hline
\end{tabular}

Aus der Tabelle eroibt sich, dass die Mäuse, die mit der Methode des erhitzten Bodens untersucht waren, bereits nach durchschnittlich drei Versuchen erlernten, das Labyrinth auf den kürzesten Wege zu durchlaufen; von den Mäusen hingegen, die mit der Methode des feuchten Bodens untersucht waren, vermochte Nr. 10 selbst nach 31 Versuchen nicht zu erlernen, das Labyrinth auf den kürzesten Wege zu durchlaufen. Die übrigen vier Mäuse erlernten dies erst uach durchschnittlich 7,5 Versuchen.

Es lässt sich hiermit behaupten, dass die Mäuse, die durch einen stärkeren Antrieb zur Ausführung einer Handlung getrieben waren, auch zweimal weniger Versuche berlurften, um eine einfache Handlung zu erlernen, als die 'Tiere, die zum Erlernen der gleichen Handlung durch einen schwächeren Antrieb getrieben waren. Diese Versuche bahnen vielleicht methodisch den Weg zur Untersuchung der Stärke verschieflener Antriebe bei einer Tierart an; sollte sich diese Methode bewähren und läge ein genügendes Versuchsmaterial vor, so wäre es vielleicht möglich, zu versuchen, die Abhängigkeit zwischen der Lern- 
geschwindigkeit und der Autriebsintensität einerseits, zwischen der Stärke von verschiedenen Autriebsqualitäten audererseits zahlenmässig zu formulieren ${ }^{1}$ ).

Ausser an erwachsenen habe ich auch an einigen noch nicht erwachsenen ganz jungen Mäusen einige Versuche angestellt.

Es stellte sich heraus, dass die drei mit der Methode des erhitżten Bodens untersuchten 'Fiere erst wach durchschnittlich 7,7 Versuchen erlernt haben, das Labyrinth auf dem kürzesten Wege zu durchlaufen (s. Tab. Nr. 11, 12, 13). Die jungen Tiere brauchten also in meinen Versuchen zweimal soviele Versuche als die erwachsenen, um die fehlerlose Ausführung der gleichen Handlung zu erlernen.

\section{Ein Fall des denkbar vollkommensten Verlaufes eines Lernvorganges.}

Den höchsten Grad der Vollkommenheit eines Lernvorganges würde ein Fall darstellen, in dem die Übung die denkbar geringste sein könnte, um eine Verknüpfung zwischen dem Reiz und der Reaktion entstehen zu lassen, d. h. diese Verknüpfung müsste bereits nach einem eiszigen Versuch zur Ausbildung gelangen. Wenn ein solcher Fall in der Natur anzutreffen wäre, so wäre es angezeigt, denselben im Gebiete kinästhetischer Reize zu suchen. Denn diese Reize, wie es jedem Forseher auf dem Gebiete der Lehre von den Erfahrungshandlungen bei Tieren auffallen muss, treten, einen genügenden Antrieb zur Ausführung der Handlung vorausgesetzt, mit der grössten Leichtigkeit in Verknüpfung mit einer bestimmten motorischen aktion ein.

Die einfachste Form, in der sich eine Verknüpfung zwischen einem kinästhetischen Reiz und einer bestimmten motorischen Reaktion würde herstellen lassen, wäre, ein Tier zwisehen rechts- bzw. linkslaufen unterscheiden zu lassen. Eine solche Untersuchung und unter dem eben erwähnten Gesichtspunkte wurde, an weissen Ratten angestellt.

1) Dass ein Zusammenhang zwischen der Antriebsstärke und Lerngeschwindiykeit auch bei den Menschen zu bestehen scheint, beweist eine von Jatz gefundene Tatsache, dass ein Kind von 21/2 Jahren zwar nicht aus einer Reibe gleichfarbiger Spielmarken, wohl aber aus einer Reihe Schokoladestückchen, von denen wie bei den Spielmarken jedes zweite festgeklebt ist, jedes zweite Stück nach kurzer Übung richtig fortnahm (cit. nach K. Bühler, Die geistige Entwicklung des Kindes, S. 92.1918. 
Die Versuchsanordnung war sehr einfach. In einem Apparate, der aus zwei quariratischen $10 \mathrm{~cm}$ hohen Seitenwänden $(x x=\mathrm{ca} .96 \mathrm{ccm}$ und $y y=74 \mathrm{ccm}$ ) ohne Boden bestand, wurde ein elektrischer Boden $D$ angebracht; bei $B$ befand sich der Wohnkäfig mit Futter und Wasser (Abb. 7).

Fine in den Vorhof $A$ gesetzte hungrige Ratte sollte nun zum Beispiel nach rechts laufen, um zum Wohnkäfig mit Futter und Wasser $(B)$ zu gelangen. Auf der "falsehen" Seite war bei $x y$ in den fünf ersten Versuchen jeder Serie eine Schiebetür aus Drahtnetz angebracht; bei den letzten fünf Versuchen jeder Serie würde die Tür entfernt und beide Gänge offen gelassen. Ausserdem erhielt die Versuchsratte, wenn sie den falschen Gang betreten hatte, einen elektrischen Schlag. Aclit Ratten (Nr. 5 und $t^{3}$ - $\delta$ sonst lauter f) wurden unter sucht; ein Versuch wurde einmal in 24 Stunden (zwischen 1-2 Uhr nachmittags) gemacht; die bisher lungrige Ratte hat in Wohnkifig $B$ Futter und Wasser vorgefunden. Das Futter war deraart normiert, dass die Ratte dasselbe in den nächsten Stunden auffrass, so dass sie bei fem nächstfolgcuflen Versuch wieder

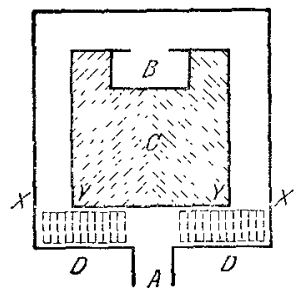

Abb. 7. A: Vorbof; $B$ : Wohnkatig; $D$ : elektrischer Boden; $C$ : toter liaum (sch affiert); bei $x y$ konnte einc Schiebetür angribacht werlen. nüchtern sein durfte. Nach jedem Versuch nit einer Ratte war der Käfig mit Seifenwasser und der elektrische Boden mit Äther wründlich gewaschen worden.

Drei Versuchsserien, von dienen je eine aus zehn Versuchen bestand, wurden mit jeder Ratte ausgeführt; in der ersten und der dritten Serie mussten die Ratten stets nach rechts, in der zweiten Serie nach links laufen, um zuin $B$ zu gelangen.

Jede nächstfolgende Serie folgte unmittelbar auf die vorangehende.

Die Resultate dieser Versuche babe ich in der Abb. 8 (S. 149) graphisch dargestellt.

Wenn wir von Nr. 2 und 4 der ersten Serie, die rufällig bein erstenmal die richtige Bewegungsrichtung eingeschlagen und bei den uächsten Versuchen dieselbe beibehalten haben, abseben, so lassen sich die Versuchsergebnisse derart zusammenfassen, dass sich drei Typen unterscheiden lassen.

Zu dem ersten Typus gehören die Ratten (Ar. 1 in allen drei 
Serien, Nr. 3 in der zweiten Serie, Nr. 6 in der ersten Serie, Nr. 8 in der ersten und der zweiten Serie), die bloss einen einzigen falschen Versuch machten, um dann bis zum Schluss der betreffenden Versuchs-
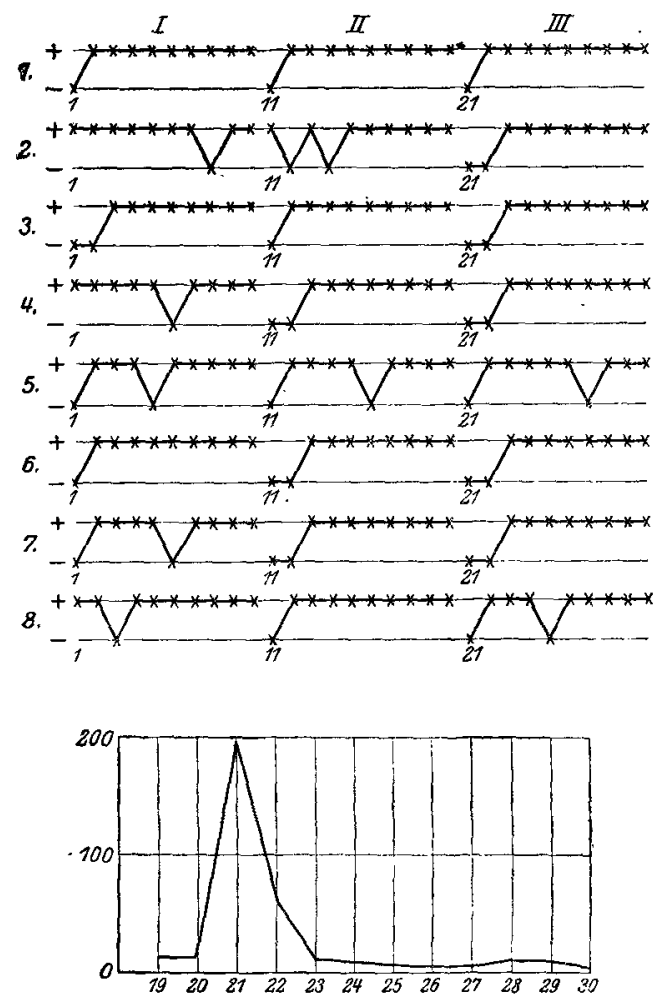

Abb. 8. In den Kurven 1 bis einscbliesslich 8 , die sich auf die $1-8$ Versuchsratten beziehen, ist auf der + Linie die richtige, auf der - Linie die falsche Bewegungsrichtung eingetragen. Die römischen Zahlen oben bezeichnen die aufeinanderfolgenden Verstichsserien: erste Serie vom 1. bis einscbliesslich 10 . Versuche, zweite Serie vom 11. bis einschliesslich 20. Versuche, dritte Serie vom 21. bis einschliesslich 30. Versuche. Die Kurve $A$ ist eine Zeitkurve, die die Durchschnittswerte für sämtliche untersuchten Tiere in der dritten Versuchsserie darstellt. Anf der Abszisse sind die Versuchstage, auf der Ordinate Sekunden eingetragen. serie stets eine richtige Bewegungsrichtung einzuschlagen. Bei diesem Typus bildet sich nach einem einzigen Versuch bereits eine relativ datrerhafte Verbindung zwischen dem Reiz und der Reaktion.

Zu dem zweiten Typus gehören die Tiere $(\mathrm{Nr} .5$ in allen drei Versuchsserien, Nr. 7 in der ersten Versuchsserie und Nr. 8 in der dritten Versuchsserie), die nach einem falschen Versuch bereits die richtige Bewegungsrichtung in den nächsten Versuchen einschlagen; nachher machen sie noch einen falschen Versuch, um dann bereits bis zum Sthluss der ganzen Versuchsserie richtig zu laufen. Bei diesem Typus entstehen also nach einem einzigen falsehen Versuch bloss kurzdauernde Verknüpfungen zwischen dem Reiz und der Reaktion.

Schliesslich gehören zu dem dritten Typus die Tiere (Nr. 2 in der zweiten und dritten Serie, Nr. 3 in der ersten und der dritten Versuchsserie, Nr. 4 in der zweiten und der dritten Serie, Nr, 6 in der zweiten und der dritten Serie, Nr. 7 in der zweiten und der dritten Serie), die nach zwei falschen Versuchen stets die richtige 
Beiträge zur Lehre von der Entstehung neuer Gewohnheiten bei den Tieren. 151

Bewegungsrichtung bis zum Schluss der ganzen Versuchsserie einschlagen. Bei diesem Typus entsteht hiermit nach bloss zwei falschen Versuchen bereits eine relativ feste Verknüpfung żwischen Reiz und Reaktion.

Dass auch die Zeit, die die Versuchsratten brauchen, um vom Vorhof bis zum Wohnkäfig zu gelangen, im Verlaufe einer Versuchsserie rapid sinkt, zeigt die Zeitkurve $A$ (in der Abb. 8), in der jch die Durchsehnittswerte von sämtlichen acht Tieren und für je einen Versuch der dritten Serie (Versuche 21 bis inkl. 30) eingetragen habe; zum Vergleich sind die gleichen Werte für die zwei letzten Versuche der zweiten Serie (Versuche 19 und 20), wäbrend welcher die Verknüpfung "nach rechts laufen" fest ausgebildet war, verzeichnet.

Als allgemeines Ergebnis dieser Versuche ist man also berechtigt, zu sagen, dass das untersuchte Verhalten der Ratten des ersten Typus den Anforderungen, die an einen denkbar vollkommensten Verlauf des Lernvorganges gestellt waren, durchaus entspricht.

Die Vollkommenheit des Lernvorganges kann nicht, wie dies in der ersten Periode der experimentellen Tierpsychologie von einigen Forschern geschah, als Maassstab der "Intelligenz" verwertet werden.

Denn es ist schwerlich zu glauben, dass die Ratten in den oben beschriehenen Versuchen sich von der Zielvorstellung leiten liessen. Die Gesch windigkeit der Ausbildung einer neuen Verknüpfung zwischen dem Reiz und der Reaktion kann nur dadurch begriffen werden, wenn man annimmt, dass eine angeborene Anlage zur Entstehung der kinestetisch-motorischen Verknüpfungen vorhanden sein musste. Die anderen Verknüpfungen bilden ja weisse Ratten ențeder gar nicht oder nur mit der grössten Langsamkeit, die beweist, dass diesen Verknüpfungen keine lebenswichtige Bedeutung zukommt, und dass sie als reine Laboratoriumsprodukte zu gelten haben. 